\title{
Community Based Distributors and Increased Ownership of the Long Lasting Insecticidal Nets in Rural Area of Jos Plateau State
}

\author{
ENVULADU E.A, BANWAT M.E, LAR L.A, ZOAKAH A. I, \\ *CORRESPONDENCE TO: ENVULADU E.A, esvula@yahoo.com,08034517244
}

Department of Community Medicine, Jos University Teaching Hospital, PMB 2076, Jos. Plateau State.

\begin{abstract}
Background: The risk of malaria infection depends on the risk of being bitten by an infected mosquito, which itself depends on levels of transmission and exposure. The risk of infection leading to severe malaria disease depends on a person's immune status and access to treatment. Long lasting insecticidal nets (LLINs) have been one of the main strategies for malaria transmission reduction. LLIns are low cost and highly effective way of reducing the incidence of malaria in people who sleep under them. By preventing malaria, LLINs reduces the need for treatment and the pressure on health services.

This study set out to assess the effectiveness of community based distribution in increasing ownership of LLIN in the community.
\end{abstract}

Methods: it was an interventional study with a study and control group using pregnant women in Dorowa Babuje as the study group and pregnant women in Vwang as the control group, baseline data was generated using an interviewer administered questionnaire in both the study and the control group. In the study group, the women were allowed to select among them those that served as community based distributors (CBDs) who were pregnant women themselves that could read and write and understood English, Hausa and the native language Birom. Subsequently, the women were sensitized about malaria, the importance of LLINs as a means of preventing malaria and how to care for the nets. The CBD then distributed the nets to the women while in the control group, after generating the data, the women were sensitized just like the study group and informed on the possible places they could obtain the LLINs but the LLIns were not distributed them. After a period of four months, using the same interviewer administered questionnaire, data was generated in both the study and the control group to compare the pre and the post intervention result and at the end of the study period, free LLINs were then distributed to the control group.

Result: Education was seen to have a statistical significant influence in the ownership of the LLINs at preintervention in both the study and the control groups. Ownership of LLINs increased statistically significantly in the study group from $9.8 \%$ to $97.6 \%(P<0.0001)$ at post-intervention. In the control group, there was no statistically significant difference in the level of ownership of LLIN at the end of the study period ( $P=0.389)$. The ownership of the LLINs was increased $89.8 \%(A F=89.8 \%)$ due to the community based distributors that shared the LLINs to the pregnant women in the community.

Conclusion: The distribution of the LLIN at the community level with the involvement of the community people has lead to the significant increase in the ownership of LLIN.

\section{Introduction}

The risk of malaria infection depends on the risk of being bitten by an infected mosquito, which itself depends on levels of transmission and exposure. The risk of infection leading to severe malaria disease depends on a person's immune status and access to treatment. Long lasting insecticidal nets (LLINs) have been one of the main strategies for malaria transmission reduction. ${ }^{1}$ LLINs are low cost and highly effective way of reducing the incidence of malaria in people who sleep under them. By preventing malaria, LLINs reduces the need for treatment and the pressure on health services. $^{2,3}$
Use of LLINs is now the central focus for the Roll Back Malaria (RBM) campaign.Targeting individual protection to vulnerable groups is an accepted priority, but community-level impacts of broader population coverage are largely ignored even though they may be just as important. ${ }^{4}$

One of the priorities of the Global strategic plan for roll back Malaria for 2005-2015 is to support countries to implement effective malaria control interventions nationwide and to give greater emphasis to community-based advocacy and social mobilization as a vital process in increasing demand for, and use of, interventions, one of which is the use of the LLINs. ${ }^{5}$ 
Community-based distribution has not been tried on a large scale, but it could possibly be added to existing strategies to successfully distribute and scale-up LLINs in rural areas. Similar strategies are being successfully used for the distribution of ivermectin for the control of onchocerciasis, filters for the control of guinea-worm and home management of malaria, one key delivery issue is scale of operation, many projects on LLINs have been implemented on a very small scale, covering individual communities or a small number of districts. Only a few LLINs delivery models have been expanded to operate at a national scale. These later models include the integration of LLINs distribution with immunization campaigns, the distribution of LLINs to ANC attendees and social marketing through public sector facilities. ${ }^{6}$

In April 2000, RBM and African Heads of States established the "Abuja targets," which include LLIN use by at least $60 \%$ and above of pregnant women and under- fives in Africa by 2005 . However, few countries have met this target, and ownership of ITNs among pregnant women in Africa is currently only $3 \%$, with rates in Nigeria reflecting this regional figure.

The determination of distribution mechanisms that will assure high coverage with the LLINs, especially in rural areas, remains a topical issue in Nigeria and in many sub-Saharan African countries (SSA). The public health care system was initially used to distribute LLINs in Nigeria, but the coverage was quite low. Currently, commercial sector distribution and social marketing of LLINs is being promoted in some states in Nigeria, but the coverage remains low. ${ }^{8}$

This study set out to assess the effectiveness of community based distribution in increasing ownership of LLIN in the community.

\section{Materials and Methods}

The study was carried out in Dorowa Babuje ward of Barkin Ladi LGA and Vwang ward in Jos South LGA in Plateau State. Dorowa Babuje is one of the twenty wards in Barkin Ladi LGA with a population of about 7,362 and an estimated population of pregnant women of 368 according to the 2006 census. Dorowa Babuje has one PHC centre, no industry with farming as the major occupation.

Vwang is one of the wards under Jos South LGA with a total population of 15,926 and estimated population of pregnant women is 426. There are two primary health care facilities and farming is the predominant occupation. ${ }^{9,10,}$

A community based interventional study was adopted with pregnant women in Dorowa Babuje as the study group while pregnant women in Vwang were the control group. The study design had both pre-intervention and post-intervention components. A minimum sample size of 117 was determined using $95 \%$ confidence level, power of $80 \%$, level of utilization of ITNs of $60 \%$ and expected level of reduction of malaria of $15 \%$ at the end of the study. A total of 174 pregnant women were studied each in the study and the control group.

The selection of the study population was by multistage sampling technique. The list of the seventeen LGAs in the state was used to select Barkin Ladi LGA and Jos South LGA by balloting.

In each of the LGAs selected, all the wards were listed out to form a sampling frame and Dorowa Babuje in Barkin Ladi was selected by balloting out of the 20 wards to serve as study group and Vwang ward in Jos South as the control group.

A list of houses containing pregnant women was drawn and a total of 216 pregnant women who fulfilled the inclusion criteria from these houses formed the sampling frame. Subsequently, from this sampling frame, the pregnant women were selected through a simple random sampling technique by balloting. In this study, we had no case of two pregnant women fulfilling the inclusion criteria coming from the same house

A semi structured interviewer administered questionnaire was administered to the pregnant women where assessment of the level of awareness, ownership and utilization of insecticide treated bed nets was made in both the study and the control groups.

The selection of the community based distributor was done by the pregnant women themselves and those selected were those who were pregnant themselves, could speak and write English and understood both Hausa and native language Birom. These CBDs went through one day sensitization training on causes and methods of prevention of malaria, treatment and retreatment of ITNs and how to mount the ITNs. The same demonstration was done for the pregnant women enlisted for the study in the community and afterwards they were asked to perform a role play to show they understood what they were taught.

After the baseline investigations was done, free insecticide treated nets (long lasting insecticidal 
nets ) which was provided by the National Roll back malaria control office Abuja were distributed to the pregnant women by the community based distributors in the study group.

At the end of four months, the same assessment was carried out in both the study and the control group and at the end of the study, the same sensitization training was given to the pregnant women in the control group and free insecticide treated nets were also distributed to them.

All data generated were processed and analyzed using the EPI info version 3.4.3 2007 statistical software. Quantitative data were described using mean, median, mode and standard deviation while chi-square was used to test for significant difference in the study and the control group. A confidence level of $95 \%$ was used and a p-value of $<0.05$ was considered significant.

Ethical clearance was obtained from the Jos University Teaching Hospital (JUTH) Ethical Committee. An advocacy was paid to the Commissioner of Health, Local Government Chairman and the village heads to inform them about the study and solicit their support. A written permission was sought and obtained from the Commissioner of Health, Local Government Chairman and the village heads of both the study and the control communities where the aims and objectives of the study was explained. A verbal consent was sought and obtained from the women before enrollment into the study.

\section{Results}

The age of the women in the study group ranged from $17-40$ years with a mean of $26 \pm 5.25$ years and $13-45$ years with a mean age of $25 \pm 5.72$ years in the control group $(\mathrm{p}=0.367)$. One hundred and seventy one $(98.3 \%)$ were married and $3(1.7 \%)$ were widowed in the study group and 173 (99.4\%) were married and $1(0.6 \%)$ was single in the control group.

In both the study and control group, majority of the women had primary school certificate, $63.8 \%$ and $64.4 \%$ in the study and control group respectively. Only $4(2.3 \%)$ of the pregnant women in the study group and $3(1.7 \%)$ in the control group had tertiary education.

Majority of the pregnant women in the study and control groups were farmers, $121(69.6 \%)$ in the study group and $105(60.3 \%)$ in the control group, others included civil servants, students, hawkers and laborers. There was no statistical significant difference between the study and the control group. $\mathrm{P}=0.625$

One hundred and sixty two (93.1\%) of the pregnant women in the study group were Christians, while 12 $(6.9 \%)$ were Muslims, in the control group, all the respondents were Christians $(\mathrm{P}<0.001)$.

Ownership of Insecticide Treated Nets was generally low at pre-intervention, of the one hundred and seventy four pregnant women interviewed in the study group, only $17(9.8 \%)$ owned ITNs and $14(8.0 \%)$ in the control group owned ITNs. Ownership of Insecticide Treated Nets improved significantly in the study group from 17 $(9.8 \%)$ to $160(97.6 \%)$ with a statistically significant increase in the level of ownership of ITNs and an attributable risk of $89.8 \%$ as a result of the intervention.

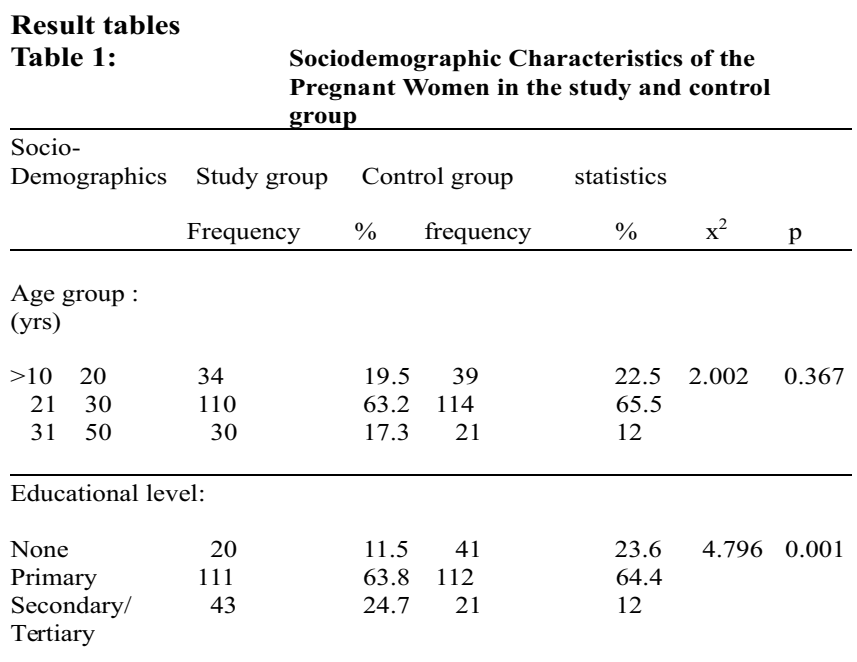

Table 2: Comparison of the level of ownership of llins at preIntervention and post-intervention

\begin{tabular}{|c|c|c|c|c|c|c|c|c|}
\hline \multicolumn{3}{|c|}{ STUDY GROUP } & \multicolumn{6}{|c|}{ CONTROL GROUP } \\
\hline \multirow[t]{2}{*}{ Ownership } & \multicolumn{2}{|c|}{ Pre-interv } & \multicolumn{2}{|c|}{ Post-interv } & \multicolumn{2}{|c|}{ Pre-interv } & \multicolumn{2}{|c|}{ Post-interv } \\
\hline & Freq & $\%$ & freq & $\%$ & freq & $\%$ & freq & $\%$ \\
\hline Yes & 17 & 9.8 & 160 & 97.6 & 14 & 8.0 & 8 & 5.1 \\
\hline No & 157 & 90.2 & 4 & 2.4 & 160 & 92.0 & 148 & 94.9 \\
\hline Total & 174 & $\mathbf{1 0 0}$ & 164 & 100 & 174 & 100 & 156 & $\mathbf{1 0 0}$ \\
\hline
\end{tabular}
$\mathrm{AR}=89.8 \%$

\section{Discussion}

This study was conducted before the nationwide mass distribution of LLIN campaign in Plateau state when the main strategy of distribution of the ITNs was through the health facilities and commercial outlets.

The findings of the study showed that as at the time when the distribution of the ITNs was going on through the health facilities, the rate of ownership of ITN was just $9.8 \%$ in the study group and $8 \%$ in the control group. When the community based 
distributors were involved in the distribution of the LLIN to the pregnant women in the study group, the rate of ownership of LLIN increased significantly from $9.8 \%$ to $97.6 \%$. The use of the community based distributors in the distribution of the LLIN brought about $89.8 \%$ increase in the ownership of LLIN in the study group compared to the control group.

According to the NDHS report of 2008, when comparing the rate of ownership of ITN, the report showed that ownership of ITN as at 2003 was 2\% which rose to only $8 \%$ in $2008 .{ }^{11}$ This survey report reveals that Nigeria is still a long way in meeting the Abuja set target of the Roll back malaria. The strategies used in the past for the distribution of ITNs was not a community based approach and that could have contributed to the low level of ownership due to the limited coverage. ${ }^{12}$

One of the strategies adopted by the Carter Center in North central Nigeria which yielded good result was the integration of ITNs distribution with Lymphatic filariasis/ onchocerciasis mass Drug administration using community directed distributors who distributed the ITNs in the community alongside the distribution of Ivermectin. This resulted in a great increase in ITN ownership among malaria-vulnerable groups over a 4-month period in two Local Government areas, Akwanga and Kanke LGA respectively, where there was an increase in the ownership of ITNs among households with under fives and pregnant women from 9 to $80 \%{ }^{13}$

In Zambia, the mass distribution of ITNs which involved distribution at community level lead to a three times increase in the level of ownership of ITNs by 2006 when compared with the level of ownership of the ITNs as at $2001^{.14}$ The result of the survey carried out in Oromia and Amhara regional states in Ethiopia in 2007 showed a 94\% ownership of ITN as against the $1.1 \%$ in 2000 and $5.7 \%$ in 2005 according to the Ethiopian Demographic and health survey following the free nets distribution to rural and poor households. ${ }^{15}$

In Uganda to achieve the national and US President's Malaria Initiative (PMI) target of $80 \%$ of pregnant women and children under-five sleeping under an ITN by 2010, one of the strategies adopted was the distribution of nets through a community based method. ${ }^{16}$

The national malaria control program in Nigeria has targeted a $50 \%$ reduction in malaria morbidity, as such in its national strategic plan, the nation plans to rapidly scale up the distribution the LLIN to every household in Nigeria and one of the channels adopted for this distribution is through a mass campaign and community based distribution in order to achieve $80 \%$ coverage of each household with at least two or more nets. ${ }^{17}$ This is a step in the right direction if followed as planned considering the benefit of the community based approach in increasing the ownership of LLINs.

\section{Conclusion}

The distribution of the LLIN at the community level with the involvement of the community people is paramount to achieving the recent target of $80 \%$ coverage of ITNs among the pregnant women set by the roll back malaria.

\section{References}

1. Jonathon L. S, Bruce A. L, Alexander Z, Sydney R. How will the reduction of tariffs and taxes on insecticide treated bed nets affect household purchases? Bulletin of the World Health Organization 2002; 80 (11):892

2. Osero J.S, Otieno M.F, Orago A.S. Maternal use of insecticide treated net in the prevention of malaria among children under five years in Nyamira district, Kenya. East African Medical Journal 2005;82(10):495500 .

3. WHO, Insecticide-treated mosquito net interventions, A manual for national control programme managers 2003 p. 5

4. Killeen G. F, Smith T.A, Heather M. F, Mshinda H, Abdullla S, Lengeler C, et al. Preventing Childhood Malaria in Africa by protecting adult from mosquitoes with insecticide treated nets. PLOS Med. 2007; 4 (7):e229.

5. Roll Back Malaria, Global Strategic plan, 2005-2015, p 3

6. Kara H, and the Tanzanian National Voucher Scheme Monitoring and Evaluation Team. Monitoring and evaluating delivery systems for Insecticide-Treated Nets: the Tanzanian national voucher scheme. December 2006.p 4

7. Olayemi I S O, Oreagba A, Mabadeje A F. Knowledge and practice of the use of insecticide Treated Nets Among Mothers in Lagos in Lagos, Nigeria (A Preliminary Survey). Nigerian Quarterly Journal of Hospital Medicine. NQJHM. 2004;14 (2): 181-184.

8. Msyamboza K, Senga E, Tetteh-Ashong E, Kazembe P, Brabin BJ. Estimation of effectiveness of interventions for malaria control in pregnancy using the screening method. : Int J Epidemiol. 2007;36(2):406-11

9. Francine N., Abdoulaye A. D, Wilfred M., thomas E., The importance and future of malaria reseach in Africa . 2004 available at http://www.ajtmh.org last accessed 17/8/09.

10. Chigozie J. U, Impact of placental plasmodium falciparum Malaria on pregnancy and perinatal outcome in sub-saharan Africa, Yale J Biol Med. 2007; $80(2): 39-44$.

11. Executive summary Netmark baseline survey on insecticide-treated materials in Nigeria, 2000. P.3 
12. Nigeria Demographic and Health Survey, Trends in net ownership and use 2008, p 192.

13. Brian G. B, Abel E, Habila G, George G, Emmanuel M, William A. H, Els Mathieu, and Frank R. Successful integration of Insecticide-Treated Bed Net distribution with mass drug administration in central Nigeria, Am.J.Trop.Med.Hyg. 2006.75(4):650-655.

14. Racheard W.S etal, National Malaria control and scaling up for impact; the Zambia experience through 2006, Am.J.Trop.Med.Hyg, 2008;79(1): 45-52
15. Baume C.A, Reithinger R, Woldehanna S, Factors associated with use and non use of mosquito nets owned in Oramia and Amhara Regional States, Ethiopia, 2009, a $\mathrm{v}$ a i 1 a b 1 e

a $\mathrm{t}$ http://www.malariajournal.com/content/8/1/264

16. Malaria Consortium, Financing and Delivering I $\mathrm{n} \mathrm{s}$ e c t i c i d e $\mathrm{T} \mathrm{e}$ a t e d $\mathrm{N}$ e $\mathrm{t} \mathrm{s}$. infomcesa@malariaconsortium.org. Available at Pub Med central. Last accessed 13/5/2008

17. FMOH/NMCP, Nigeria strategic plan 2009-2013, pp 17-20. 